\title{
Perímetro escrotal e características seminais de touros da raça Nelore selecionados para precocidade sexual
}

\author{
Scrotal perimeter and seminal characteristics of Nelore bulls selected \\ for sexual precocity \\ F.P.C. Lima, P.R. Xavier, J.A.G. Bergmann, A.P. Marques Júnior* \\ Escola de Veterinária - Universidade Federal de Minas Gerais - Belo Horizonte, MG

\section{RESUMO} \\ Vinte e quatro touros jovens da raça Nelore foram distribuídos em grupo 1 (G1, à puberdade) e grupo 2 \\ (G2, pós-puberdade), segundo a concentração seminal e motilidade progressiva dos espermatozoides. \\ Características de produção, seminais e endócrinas foram avaliadas à puberdade e pós-puberdade, aos 12 , \\ 14, 16 e 18 meses de idade. A média de idade, de peso e de perímetro escrotal à puberdade foram,

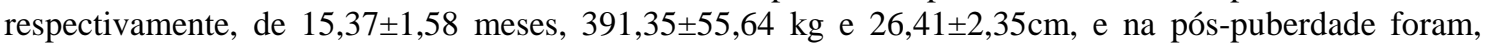 \\ respectivamente, de 17,49 $\pm 1,73$ meses; $451,75 \pm 35,44 \mathrm{~kg}$ e $29,93 \pm 2,59 \mathrm{~cm}$. As características seminais na \\ pós-puberdade aumentaram em relação à puberdade. Pode-se inferir que os tourinhos da raça Nelore \\ submetidos a um programa de seleção para precocidade sexual manifestaram a puberdade em torno de 15 \\ meses de idade e que touros jovens com maior perímetro escrotal apresentaram menor idade à puberdade.
}

Palavras-chave: touro, Nelore, puberdade, sêmen

\begin{abstract}
Twenty-four young Nelore bulls were divided in group 1 (G1, at puberty) and group 2 (G2, after puberty), according to characteristics of seminal concentration and gradual motility of the spermatozoa. Zoothecnic, seminal and endocrine characteristics were evaluated at puberty and after puberty, at 12, 14, 16 and 18 months of age. The average of age, weight and scrotal perimeter at puberty were, respectively, $15,37 \pm 1,58$ months, 391,35 $\pm 55,64$ and $26.41 \pm 2,35 \mathrm{~cm}$, and in the period after puberty they were,

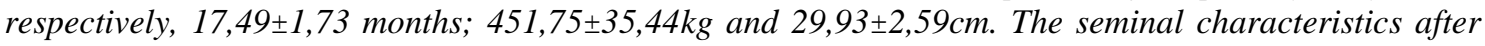
puberty increased in relation to that at puberty. It is concluded that the young Nelore bulls submitted to one program of election for sexual precocity reached puberty around 15 months of age, and young bulls with higher escrotal perimeter had puberty at an early age.
\end{abstract}

Keywords: Nelore, puberty, bulls, sperm

\section{INTRODUÇÃO}

Para avaliar a capacidade reprodutiva do touro, têm sido propostas várias características envolvendo medidas testiculares e qualidade do sêmen (Fonseca et al., 1997). Entre as características propostas, o exame andrológico, que incluiu concentração, motilidade e morfologia dos espermatozoides no ejaculado, e as medidas do perímetro escrotal (PE) têm sido os mais utilizados por estarem correlacionados

Recebido em 6 de setembro de 2012

Aceito em 2 de julho de 2013

*Autor para correspondência (corresponding author)

E-mail: ampinho@ufmg.br com a fertilidade em touros e pela praticidade e facilidade de serem realizados (Almquist e Amann, 1976; Lunstra et al., 1978).

A puberdade é o período em que se verifica rápido desenvolvimento reprodutivo. A aplicação de conhecimentos referentes a essa fase possibilita a seleção de reprodutores mais precoces. Existem pesquisas sobre o desenvolvimento reprodutivo puberal de bovinos de raças taurinas em ambiente de clima temperado, mas poucas abordaram o tema em 
raças zebuínas no Brasil (Godinho e Cardoso, 1984; Dode et al., 1989; Freneau, 1996; Devkota et al., 2008). A puberdade caracteriza-se como a idade em que ocorre rápido crescimento testicular, mudanças na secreção de hormônio luteinizante e testosterona e, como consequência, início da espermatogênese (Almquist e Amann, 1976).

Segundo Wolf et al. (1965), as características dos ejaculados na fase puberal apresentam grande variabilidade qualitativa e quantitativa. Para uma avaliação adequada da capacidade reprodutiva do touro, é necessário considerar a idade do animal no momento da coleta, além de outras características, como peso corporal, raça, alimentação e composição genética (Lunstra et al., 1978).

Segundo Vale Filho et al. (2001), tourinhos são classificados como precoces quando, aos 12 meses, forem observados espermatozoides em seus ejaculados, mesmo com baixa motilidade ( 5 a $10 \%$ ) e concentração de 25 a $50 \times 10^{6} \mathrm{sptz} / \mathrm{mL}$ por ejaculado e PE entre 24 e $26 \mathrm{~cm}$; e superprecoces aqueles que, nessa mesma idade, apresentarem motilidade espermática progressiva variando de 20 a $30 \%$ e concentração de $50 \times 10^{6}$ sptz/mL por ejaculado, além de PE igual ou superior a $26 \mathrm{~cm}$. Por isso, a avaliação de sêmen de touros mais novos pode ser um método auxiliar na identificação de animais sexualmente precoces (Siddiqui et al., 2008).

O objetivo deste trabalho foi acompanhar o desenvolvimento testicular de animais submetidos a um programa de seleção para precocidade sexual, em diferentes idades, e avaliar características seminais à puberdade e pós-puberdade.

\section{MATERIAL E MÉTODOS}

Vinte e quatro tourinhos da raça Nelore (Bos taurus indicus) foram divididos em dois grupos, G1 (à puberdade) e G2 (pós-puberdade), segundo características de concentração seminal e motilidade progressiva dos espermatozoides (Wolf et al., 1965). Para determinar a puberdade, consideraram-se aqueles que apresentaram ejaculado com $50 \times 10^{6}$ espermatozoides por $\mathrm{mL} \mathrm{e}$ $10 \%$ de motilidade mínima, e, para a póspuberdade, aqueles que, independentemente de idade e peso, apresentaram valores da concentração e motilidade seminal mais altos que os estabelecidos para definir animais à puberdade.

As características avaliadas na pós-puberdade incluíram as mensurações posteriores à mensuração no momento da puberdade. Os animais foram mantidos nas mesmas condições ambientais e de manejo desde o nascimento e os grupos, separados em piquetes, receberam silagem de milho com concentrado no cocho. Características zootécnicas, seminais e endócrinas foram avaliadas à puberdade $\mathrm{e}$ pós-puberdade e também em idades pré-estabelecidas, aos 12, 14, 16 e 18 meses. As coletas de sêmen foram realizadas em intervalos de 60 dias, dos 12 aos 18 meses, pelo método da eletroejaculação. A avaliação física do sêmen volume, motilidade, vigor e concentração espermáticos - foi realizada imediatamente após as colheitas, na própria fazenda, e as avaliações morfológicas - defeitos maiores e menores foram feitas após fixação do sêmen em solução de formol salina tamponada (Hancock, 1957), no laboratório da Alta Genetics, localizado em Uberaba, MG. Foi feita análise de variância (ANOVA), e as médias foram comparadas pelo teste F ao nível de 5\% (Sampaio, 2002).

\section{RESULTADOS E DISCUSSÃO}

Os tourinhos avaliados apresentaram desenvolvimento corporal maior que o de animais mantidos nas mesmas condições de manejo e alimentação, conforme demonstrado pelo peso corporal e perímetro escrotal aferidos aos 12, 14, 16 e 18 meses de idade (Tab. 2). Houve diferença entre as características de crescimento corporal e testicular, e os aspectos físicos e morfológicos dos ejaculados, ao longo do período experimental, são apresentados na Tab. 2. Houve diferenças entre essas características nos dois momentos de avaliação (Tab. $1 ; \mathrm{P}<0,05)$. 
Tabela 1. Idade, peso corporal, perímetro escrotal e características seminais de touros da raça Nelore $(n=24)$ à puberdade e pós-puberdade, submetidos a um programa de seleção para precocidade sexual

\begin{tabular}{lcc}
\hline \multicolumn{1}{c}{ Características } & A Puberdade & Pós-Puberdade \\
\hline Idade (meses) & $15,37 \mathrm{~B} \pm 1,58$ & $17,49 \mathrm{~A} \pm 1,73$ \\
Peso corporal $(\mathrm{kg})$ & $391,35 \mathrm{~B} \pm 55,64$ & $451,75 \mathrm{~A} \pm 35,44$ \\
Perímetro escrotal $(\mathrm{cm})$ & $26,41 \mathrm{~B} \pm 2,35$ & $29,93 \mathrm{~A} \pm 2,59$ \\
Volume de sêmen $(\mathrm{mL})$ & $3,47 \mathrm{~A} \pm 1,95$ & $2,55 \mathrm{~B} \pm 1,46$ \\
Motilidade espermática $(\%)$ & $0,37 \mathrm{~B} \pm 0,23$ & $0,63 \mathrm{~A} \pm 0,29$ \\
Vigor espermático (0-5) & $0,50 \mathrm{~B} \pm 0,14$ & $0,71 \mathrm{~B} \pm 0,23$ \\
Concentração espermática $\left(\mathrm{x} 10^{6} \mathrm{~mL}\right)$ & $560,05 \mathrm{~B} \pm 314,27$ & $938,02 \mathrm{~A} \pm 362,84$ \\
Defeitos maiores $(\%)$ & $22,58 \mathrm{~A} \pm 14,85$ & $15,0 \mathrm{~B} \pm 15,68$ \\
Defeitos menores $(\%)$ & $17,75 \mathrm{~A} \pm 14,68$ & $8,66 \mathrm{~B} \pm 9,37$
\end{tabular}

Valores com letras diferentes nas linhas diferem entre si $(\mathrm{P}<0,05)$.

Tabela 2. Média total da idade, G1 (puberdade) + G2 (pós-puberdade), peso corporal e características seminais aos 12, 14, 16 e 18 meses de idade em touros da raça Nelore $(n=24)$, submetidos a um programa de seleção para precocidade sexual

\begin{tabular}{lcccc}
\hline Características & \multicolumn{3}{c}{ Idade } \\
\hline & 12 meses & 14 meses & 16 meses & 18 meses \\
Idade real (meses) & $13,48 \mathrm{D}$ & $15,51 \mathrm{C}$ & $17,68 \mathrm{~B}$ & $19,61 \mathrm{~A}$ \\
Peso corporal $(\mathrm{kg})$ & $331,6 \mathrm{D}$ & $423,3 \mathrm{C}$ & $446,6 \mathrm{~B}$ & $487,3 \mathrm{~A}$ \\
Perímetro escrotal $(\mathrm{cm})$ & $23,37 \mathrm{C}$ & $28,43 \mathrm{~B}$ & $29,45 \mathrm{AB}$ & $30,77 \mathrm{~A}$ \\
Volume de sêmen $(\mathrm{mL})$ & $2,45 \mathrm{~A}$ & $1,36 \mathrm{~B}$ & $3,20 \mathrm{~B}$ & $3,37 \mathrm{~B}$ \\
Motilidade espermática $(\%)$ & $0,20 \mathrm{~B}$ & $0,56 \mathrm{~A}$ & $0,62 \mathrm{~A}$ & $0,60 \mathrm{~A}$ \\
Vigor espermático (0-5) & $0,35 \mathrm{~B}$ & $0,63 \mathrm{~A}$ & $0,69 \mathrm{~A}$ & $0,65 \mathrm{~A}$ \\
Concentração espermática $\left(\mathrm{x} 10^{6} \mathrm{~mL}\right)$ & $204,7 \mathrm{~B}$ & $925,9 \mathrm{~A}$ & $996,8 \mathrm{~A}$ & $962,3 \mathrm{~A}$ \\
Defeitos maiores $(\%)$ & $20,79 \mathrm{~A}$ & $17,47 \mathrm{~A}$ & $16,42 \mathrm{~A}$ & $13,42 \mathrm{~B}$ \\
Defeitos menores (\%) & $29,6 \mathrm{~A}$ & $10,7 \mathrm{~B}$ & $8,0 \mathrm{~B}$ & $5,7 \mathrm{~B}$ \\
\hline
\end{tabular}

Valores com letras diferentes nas linhas diferem entre si $(\mathrm{P}<0,05)$.

Quanto à avaliação física do sêmen (Tab. 2), observou-se aumento progressivo nos valores até o final do experimento $(\mathrm{P}<0,05)$, indicando que os animais, à medida que adquiriam maturidade sexual, apresentavam melhora na qualidade seminal. Alterações na morfologia espermática defeitos espermáticos maiores e menores apresentaram queda contínua ao longo das observações $(\mathrm{P}<0,05)$, o que está relacionado com a maturação do epitélio seminífero e progressiva estabilização das características seminais.

Os tourinhos apresentaram aumento no peso corporal e no PE como esperado e de acordo com a literatura (Coulter, 1986). O significativo aumento do PE na pós-puberdade, quando comparado ao da puberdade, indica que o perímetro escrotal continua a crescer mesmo após a puberdade. No presente trabalho, a idade média em que os animais alcançaram a puberdade foi de 15,4 meses (Tab. 1), semelhante ao observado por Freneau et al. (2006), que utilizaram animais da raça Nelore criados em condições de pastagem. Em animais europeus, Bos taurus taurus, a puberdade foi atingida aos 10,2 meses de idade, com o PE médio de $27,8 \mathrm{~cm}$ (Lunstra et al., 1978), sugerindo que eles sejam mais precoces do que os animais Bos taurus indicus. Fisiologicamente, esses dados são confirmados pela literatura, que mostra precocidade de crescimento corporal e, consequentemente, do sistema neuroendócrino de animais europeus. Segundo os valores médios para idade à puberdade, pode-se sugerir que a idade à puberdade na raça Nelore tem diminuído ao longo dos anos, provavelmente em razão do melhoramento genético e contínua seleção de animais precoces, o que tem sido feito por criadores especializados. Comportamento semelhante foi observado também nos trabalhos que utilizaram animais criados em condições de pastagens, salientando, contudo, que essas 
características são muito variáveis e dependentes das condições ambientais e de manejo de cada sistema.

As características seminais na pós-puberdade (Tab. 1) aumentaram em relação à puberdade. Motilidade, vigor e concentração espermáticos aumentaram, semelhantemente ao encontrado por Devkota et al. (2008), e indicaram gradual desenvolvimento reprodutivo e alcance da maturidade sexual. Como esperado, o número de defeitos espermáticos diminuiu com a progressiva estabilização das características seminais após a puberdade, até o animal atingir a maturidade sexual. Nos animais entre 12 e 14 meses de idade, ou seja, na faixa etária que alcançam a puberdade (Tab.1 e 2), constatou-se maior taxa de patologias espermáticas - defeitos maiores e menores - do que nos animais póspúberes. A presença de patologias espermáticas, nessa fase, pode ser atribuída ao estado de imaturidade do epitélio seminífero e dos espermatozoides, que ainda estão em processo de formação e aquisição de estabilidade (Barth, 2004).

Os animais deste experimento atingiram os patamares de qualidade do sêmen sugeridos por Chenoweth e Spitzer (1992), com máximo de $30 \%$ de patologias espermáticas. A idade do touro por ocasião da colheita de sêmen afeta as características seminais (Fuerst-Waltl et al., 2006). A literatura menciona que, na avaliação dessas características, elas tendem a melhorar à medida que o animal alcança a maturidade sexual (Almquist e Amann, 1976).

Vale Filho (1989) afirmou que, na avaliação do sêmen, admite-se até $15 \%$ de defeitos maiores e até $25 \%$ de defeitos menores, não podendo a soma total dos dois tipos de defeitos ultrapassar $30 \%$. Os animais avaliados neste experimento estão dentro dos padrões propostos, indicando bom desenvolvimento reprodutivo, mesmo considerando a idade precoce em que foi efetuada a avaliação.

As características seminais apresentaram elevado desvio-padrão, justificado pelas diferenças na precocidade sexual entre os animais. Esse achado é característico na fase puberal de desenvolvimento reprodutivo em touros, pois os animais apresentam epitélio seminífero ainda em estruturação (Amann, 1983). Os resultados aqui observados assemelham-se aos relatados para tourinhos de raças taurinas e mestiços, quanto à baixa qualidade dos ejaculados, próprios da fase puberal na espécie bovina. Esse fato deveria ser considerado na avaliação de touros jovens, nessa fase, para a identificação de indivíduos precoces, e não como critério de descarte.

No presente estudo, houve variação no volume seminal entre os tratamentos, o que se justifica, pois o volume de sêmen pode variar de acordo com o método usado para coleta, cerca de 2 a $6 \mathrm{~mL}$ por meio da vagina artificial e até $25 \mathrm{~mL}$ pelo método de eletroejaculação. No entanto, a variação depende do animal, da eficiência da contração dos vasos deferentes e cauda do epidídimo e da resposta aos estímulos (Lunstra et al., 1978; Makarechian et al., 1985).

Os tourinhos avaliados neste estudo podem ser considerados precoces, apresentando $\mathrm{PE}$ à puberdade de $26,4 \mathrm{~cm}$, em relação ao encontrado por Silva et al. (2002) para animais da raça Nelore. Em estudo realizado por Brito et al. (2004), os animais foram divididos em precoces e tardios, com PE médio de $22,5 \mathrm{~cm}$ para animais precoces. Freneau et al. (2006) observaram, em estudo com animais em pasto, PE médio à puberdade de $21,7 \mathrm{~cm}$. Essas diferenças observadas podem ser atribuídas à maior disponibilidade de alimentos e qualidade nutricional, à maior precocidade pela seleção de touros doadores do sêmen utilizados na produção dos animais desse experimento, ou por se tratar de animais de elite (PO), produtos de programas de melhoramento genético adotados na fazenda.

Observa-se que a puberdade em tourinhos da raça Nelore pode ser predita utilizando-se características zootécnicas, como a idade, o peso corporal e o perímetro escrotal, e a associação dessas características com a avaliação seminal pode se constituir em medida auxiliar na avaliação da precocidade sexual. Nas condições em que este estudo foi conduzido, conclui-se que os tourinhos da raça Nelore submetidos a um programa de seleção para precocidade sexual manifestaram a puberdade em torno de 15 meses de idade e com PE de $26,4 \mathrm{~cm}$. 


\section{CONCLUSÃO}

Touros jovens com maior perímetro escrotal apresentaram menor idade à puberdade, e sua associação com os padrões seminais do início da puberdade à maturidade sexual pode auxiliar a seleção de animais geneticamente mais precoces.

\section{REFERÊNCIAS}

ALMQUIST, J.O.; AMANN, R.P. Reproductive capacity of dairy bulls. Puberal characteristics and postpuberal changes in production of semen and sexual activity of Holstein bulls ejaculated frequently. J. Dairy Sci., v.59, p.986-991, 1976.

AMANN, R.P. Endocrine changes associated with onset of spermatogenesis in Holstein bulls. J. Anim. Sci., v.66, p.2606-2622, 1983.

BARTH, A.D. Pubertal development of Bos taurus beef bulls. In: WORLD BUIATRICS CONGRESS, 23., 2004. Quebec. Proceedings..., Quebec, 2004. (CD ROM).

BRITO, L.F.C.; SILVA, A.E.D.F.; UNANIAN, M.M. et al. Sexual development in early and late maturing Bos indicus and Bos indicus $\mathrm{x}$ Bos taurus crossbred bulls in Brazil. Theriogenology, v.62, p.1198-1217, 2004.

CHENOWETH, P.J.; SPITZER, J.C. A new bull breeding soundness evaluation form. Proc. Society of Theriogenology, AGM, p.63-70, 1992.

COULTER, G.H. Puberty and postpuberal development of beef bulls. In: MORROW, D.A. Current therapy in Theriogenology. 2nd ed. Philadelphia: Saunders co., 1986. p.142-148.

DEVKOTA, B.; KOSEKI, T.; MATSUI, M. et al. Relationships among age, body weight, scrotal circumference, semen quality and pheriperal testosterone and estradiol concentrations in pubertal and postbubertal Holstein bulls. J. Vet. Med. Sci., v.70, p.119-121, 2008.

DODE, M.A.N.; SCHENK, J.A.P.; SILVA, A.E.D.F. Determinação da puberdade em machos Nelore e Mestiços. Rev. Bras. Reprod. Anim., v.14, p.227-233, 1989.

FONSECA, V.O.; SANTOS, N.R; MALINSKI, P.R. Classificação andrológica de touros zebus (Bos taurus indicus) com base no perímetro escrotal e características morfo-físicas do sêmen. Rev. Bras. Reprod. Anim., v.21, p.36-39, 1997.
FRENEAU, G.E. Biopsia testicular aberta efetuada na puberdade e na pós-puberdade $e$ suas consequências sobre a gametogênese $e$ o sêmen de touros Nelore. 167f., 2006. (Tese de Doutorado). Escola de Veterinária, UFMG, Belo Horizonte.

FRENEAU, G.E.; VALE FILHO, V.R.; MARQUES Jr. et al. Puberdade em touros Nelore criados em pasto no Brasil: características corporais testiculares e seminais e de índice de capacidade andrológica por pontos. Arq. Bras. Med. Vet. Zootec, v.58, p.1107-1115, 2006.

FUERST-WALTL, B.; SCHWARZENBACHER, H.; PERNER, C.; SOLKNER, J. Effects of age and environmental factors on semen production and semen quality of Austrian Simmental bulls. Anim. Reprod. Sci., v.95, p.27-37, 2006.

GODINHO, H.P; CARDOSO, F.M. Gonadal and extragonadal sperm reserves of the brazilian Nelore Zebu (Bos indicus). Andrologia, v.16, p.131-134, 1984.

HANCOCK, J.L. The morfology of boar spermatozoa. J. R. Microsc. Soc., v.76, p.84-97, 1957.

LUNSTRA, D.D.; FORD, J.J.; ECHTERNKAMP, S.E. Puberty in beef bulls hormone concentrations, growth, testicular development, sperm production and sexual agressiveness in bulls of different breeds. J. Anim. Sci., v.46, p.1054-1062, 1978.

MAKARECHIAN, M.; FARID, A.; BERG, RT. Scrotal circumference, semen characteristics, growth parameters and their relationships in young beef bulls. Can. J. Anim. Sci., v.65, p.789$798,1985$.

SAMPAIO, I.B.M. Estatística aplicada à experimentação animal. 2.ed. Belo Horizonte: FEP-MVZ. 2002. p.265.

SIDDIQUI, M.A.R.; BHATTACHARJJE, J.; ZAS, Z.C.; ISLAM, M.M. et al. Crossbred bull selection for bigger scrotum and shorter age at puberty with potentials for better quality semen. Reprod. Dom. Anim., v.43, p.74-79, 2008.

SILVA, A.E.D.F.; UNANIAN, M.M.; CORDEIRO, C.M.T. et al. Relação da perímetro escrotal e parâmetros da qualidade de sêmen em touros da raça Nelore, PO. Rev. Bras. Zootec., v.31, p.1157-1165, 2002. 
VALE FILHO, V.R. Padrões de sêmen bovino, para o Brasil. Análise e sugestões. ln: CONGRESSO BRASILEIRO DE REPRODUÇÃO ANIMAL, 8., 1989. Belo Horizonte. Anais... Belo Horizonte: CBRA, 1989. p.94-118.

VALE FILHO, V.R.; ANDRADE, V.J.; QUIRINO, C.R. et al. Perfil andrológico de touros da raça Tabapuã (Bos taurus indicus) de um a dois anos de idade, criados extensivamente nos estados de Minas Gerais, Bahia e Espírito Santo, Brasil. In: CONGRESSO BRASILEIRO DE REPRODUÇÃO ANIMAL, 14., 2001. Belo Horizonte. Anais ... Belo Horizonte: CBRA, 2001.

WOLF, F.R.; ALMQUIST, J.O.; HALE, E.B. Prepuberal behavior and puberal characteristics of beef bulls on high nutrient allowance. J. Anim. Sci., v.24, p.761-765, 1965. 